Pacific Journal of Mathematics

DECOMPOSITIONS OF ALGEBRAICALLY COMPACT 


\title{
DECOMPOSITIONS OF ALGEBRAICALLY COMPACT MODULES
}

\author{
Alberto FACCHINI
}

\begin{abstract}
This paper is mainly concerned with describing the category of all algebraically compact (= pure-injective) modules. A family of functors from this category to categories of injective modules, that is, spectral categories, is defined. Via these functors we transfer the decompositions of the objects of a spectral category and their invariants to algebraically compact modules. For instance, as a corollary we find the decompositions and the invariants for algebraically compact abelian groups and the decompositions for algebraically compact modules over Prüfer rings. Our results yield a connection between the theory of algebraically compact modules and the one of injective modules.
\end{abstract}

The theory of algebraically compact abelian groups, mainly due to Kaplansky [12], Loś [13], Balcerzyk [2] and Maranda [14], has been extended to modules by Stenström [18] and Warfield [19]. Recently the structure of algebraically compact modules has been studied with methods of model theory by Fisher [6], Garavaglia [9], [10], and Ziegler [21]. It was Maranda [14] who first pointed out the similarity between the theory of algebraically compact abelian groups and that of divisible (injective) groups. Later Warfield implicitly asked in the last paragraph of [20] whether it was possible to fit his methods and results on injective modules to algebraically compact ones.

In this paper we answer Warfield's question and study algebraically compact modules in the context of categories of injectives, i.e. spectral categories. Spectral categories (Grothendieck categories in which every exact sequence splits) were introduced by Gabriel and Oberst [8] and studied by Roos [17], Goodearl and Boyle [11], where a beautiful dimension theory for the objects of a spectral category was constructed, and the author [5].

After the preliminaries of the first section, we define a family of functors $F_{E}$ from the full subcategory of Mod- $R$ generated by all algebraically compact modules into suitable spectral categories. This is done in section two by using the powerful results on algebraically compact modules obtained by Zimmermann-Huisgen and Zimmermann in [22]. In section three we show that our functors $F_{E}$ map pure-injective envelopes 
of direct sums into injective envelopes of direct sums. In section four an additive category whose objects are algebraically compact modules and whose small full subcategories are equivalent to full subcategories of spectral categories is constructed. Section five is devoted to the decomposition of algebraically compact modules into five types- $\mathrm{I}_{f}, \mathrm{I}_{\infty}, \mathrm{II}_{f}$, II $_{\infty}$, III-in analogy with von Neumann's and Murray's classification.

Finally, section six shows the existence of a close relation between dimension theory for nonsingular injective modules constructed by Goodearl and Boyle [11], the dimensions for algebraically compact modules defined by Ziegler [21], and the invariants for algebraically compact abelian groups considered in Orsatti's book [16].

1. Preliminaries. All rings in this paper are associative with 1 and all modules are right unital modules unless otherwise specified. Endomorphism rings act on the left of their modules. For any ring $R$, Mod- $R$ denotes the category of all right $R$-modules. We write the cardinality of a set $S$ as $|S|$. Given a set $S$ and a module $M, M^{(S)}$ and $M^{S}$ denote the direct sum and the direct product, respectively of $|S|$ copies of $M$. A module $M$ is algebraically compact (abbreviate a.c. in the proofs) if every finitely soluble family of linear equations over $R$ in $M$ has a simultaneous solution. A submodule $A$ of a right $R$-module $B$ is pure if for any left $R$-module $F$ the natural homomorphism $A \otimes F \rightarrow B \otimes F$ is injective. A module $M$ is pure-injective if for any module $B$ and pure submodule $A$ of $B$, any homomorphism of $A$ into $M$ can be extended to a homomorphism of $B$ into $M$. A module is pure-injective if and only if it is algebraically compact [19, Thm. 2].

For every left $R$-module $M$ we denote by $M^{*}$ its dual $\operatorname{Hom}_{Z}(M, Q / Z)$, where $Z$ is the integers and $Q$ the rationals. The group $M^{*}$ may be considered as a right $R$-module by defining $(\varphi r)(x)=\varphi(r x)$ for $x \in M$, $r \in R$ and $\varphi \in M^{*}$. Similarly for right $R$-modules.

Our study of algebraically compact (= pure-injective) modules is based on the following Theorem and its Corollary.

THEOREM 1. Let $R$ be a ring. Then there exists an algebraically compact right $R$-module $E$ such that every right $R$-module $M$ is isomorphic to a pure submodule of $E^{2^{|M|}}$.

Proof. The proof is modelled on the proof of [4, Thm. 2.4]. Let $\mathscr{F}$ denote a set of representatives of the left finitely generated $R$-modules. Then the right $R$-module $E=\left(\oplus_{F \in \mathscr{F}} F\right)^{*}$ is a.c. by [18, Prop. 9.1 and 9.2]. Let $M$ be a right $R$-module and $\left\{M_{i} \mid i \in I\right\}$ be the family of all 
finitely generated submodules of the left $R$-module $M^{*}$, so that $|I| \leq 2^{|M|}$. By [4, pg. 201, Lemme], the canonical epimorphism $\oplus_{i \in I} M_{i} \rightarrow M^{*}$ has a pure kernel, and therefore the dual morphism $M^{* *} \rightarrow\left(\bigoplus_{i \in I} M_{i}\right)^{*}$ is split mono [18, Prop. 9.1]. By [4, Prop. 2.2(a)] $M$ is a pure submodule of $M^{* *}$. Since $\left(\oplus_{i \in I} M_{i}\right)^{*}$ is a direct summand of $E^{I}$, it follows that $M$ is isomorphic to a pure submodule of $E^{I}$.

COROllaRY 1. Let $R$ be $a$ ring and let $\alpha$ be a cardinal number. Then there exists a right $R$-module $E$ with the following properties:

(a) $E$ is algebraically compact;

(b) every algebraically compact right $R$-module of cardinality $\leq \alpha$ is isomorphic to a direct summand of $E$;

(c) every algebraically compact right $R$-module $M$ is isomorphic to a direct summand of $E^{2^{|M|}}$.

Proof. The module $E^{2^{\alpha}}$ of Theorem 1 has the desired properties.

2. Definition of the functors and their properties. Fix an algebraically compact right $R$-module $E$ with the properties of Corollary 1 . Let $A=\operatorname{End}_{R}(E)$ be its endomorphism ring, so that $E$ has an $(A, R)$-bimodule structure. Let $H_{E}$ denote the covariant functor $\operatorname{Hom}_{R}(E,-)$ : Mod-R $\rightarrow$ Mod-A. If $J(A)$ is the Jacobson radical of $A$, set $\bar{A}=A / J(A)$. Recall that for any right $A$-module $N$ the reject of $\bar{A}$ in $N$ is defined by $\operatorname{Rej}_{N}(\bar{A})=\bigcap\left\{\operatorname{ker} h \mid h \in \operatorname{Hom}_{A}(N, \bar{A})\right\}$; it is the unique smallest submodule $K$ of $N$ such that $N / K$ is cogenerated by $\bar{A}[1, \S 8]$. Define $R_{E}(N)=N / \operatorname{Rej}_{N}(\bar{A})$, so that $R_{E}(N)$ is an object of Mod- $\bar{A}$. If $f: N \rightarrow P$ is a homomorphism, $f\left(\operatorname{Rej}_{N}(\bar{A})\right) \leq \operatorname{Rej}_{P}(\bar{A})$, so that $f$ induces a homomorphism $R_{E}(f): R_{E}(N) \rightarrow R_{E}(P)$. Clearly $R_{E}$ is an additive functor $\operatorname{Mod}-A \rightarrow \operatorname{Mod}-\bar{A}$. Let $F_{E}=R_{E} H_{E}: \operatorname{Mod}-R \rightarrow \operatorname{Mod}-\bar{A}$ denote the composite functor. Note that $F_{E}(E)=\bar{A}$.

LEMMA 1. For every algebraically compact right $R$-module $M, F_{E}(M)$ is a nonsingular injective right module over the regular, right self-injective ring $\bar{A}=A / J(A)$.

Proof. The ring $\bar{A}=A / J(A)$ is regular and right self-injective by [22, Thm. 9]. Since $F_{E}(M)=R_{E} H_{E}(M)$ is cogenerated by $\bar{A}, F_{E}(M)$ is a nonsingular right $\bar{A}$-module. In order to prove that $F_{E}(M)$ is injective, it is sufficient to prove that $R_{E}\left(A^{I}\right)$ is injective for every power $A^{I}$ of $A$, because $M$ is a direct summand of a power of $E, H_{E}$ respects direct products and $H_{E}, R_{E}$ are additive functors. Note that $A$, and therefore $A^{I}$, 
are a.c. [19, Prop. 7]. The proof that $R_{E}\left(A^{I}\right)$ is an injective $\bar{A}$-module is modelled on the proof of [22, Thm. 9(2)]. Given a homomorphism $f$ from a right ideal $K$ of $\bar{A}$ into $R_{E}\left(A^{I}\right)$, we choose a family of idempotents $\left\{\varepsilon_{l} \mid i \in I\right\}$ of $\bar{A}$ such that $\oplus_{l \in I} \varepsilon_{l} \bar{A}$ is essential in $K$ (possible, because $\bar{A}$ is regular). Since $R_{E}\left(A^{I}\right)$ is nonsingular, it is enough to extend the restriction $f_{1}: \oplus_{i \in I} \varepsilon_{i} \bar{A} \rightarrow R_{E}\left(A^{I}\right)$ of $f$ to all of $\bar{A}$. By [22, Thm. 9(1) and Lemma 13] there are idempotents $e_{l}$ of $A$ such that (1) $e_{i}+J(A)=\varepsilon_{l}$, (2) the sum of the $e_{i} A$ is again direct, and (3) every finite subsum is a direct summand of $A$. In particular, $\oplus_{i \in I} e_{l} A$ is a pure, projective right ideal of $A$. Using its projectivity we find a homomorphism $f_{2}: \bigoplus_{i \in I} e_{l} A \rightarrow A^{I}$ with $f_{1} \pi_{1}=\pi_{2} f_{2}$, where $\pi_{1}: \bigoplus_{l \in I} e_{i} A \rightarrow \oplus_{l \in I} \varepsilon_{l} \bar{A}$ and $\pi_{2}: A^{I} \rightarrow R_{E}\left(A^{I}\right)$ are the canonical projections. Since $\oplus_{l \in I} e_{l} A$ is pure in $A$ and $A^{I}$ is pure-injective, the homomorphism $f_{2}$ can be extended to $f_{3}: A \rightarrow A^{I}$. The homomorphism $R_{E}\left(f_{3}\right): \bar{A} \rightarrow R_{E}\left(A^{I}\right)$ coincides with $f$ on $\oplus_{l \in I} \varepsilon_{i} \bar{A}$.

Definition. For any ring $S$, we use $\mathscr{A}(S)$ and $\mathscr{N}(S)$ to denote the full subcategories of Mod-S generated by all algebraically compact right $S$-modules and all nonsingular injective right $S$-modules respectively.

Recall that $\mathscr{N}(S)$ is a spectral category, that is an abelian category with exact direct limits and a generator in which every exact sequence splits [11, Prop. 1.13]. Conversely, a category is spectral if and only if it is equivalent to the category $\mathscr{N}(S)$ for some regular, right self-injective ring $S$ [11, Thm. 1.14]. Lemma 1 immediately yields the following

THEOREM 2. The functor $F_{E}:$ Mod- $R \rightarrow$ Mod- $\bar{A}$ induces a functor of the category $\mathscr{A}(R)$ into the spectral category $\mathscr{N}(\bar{A})$.

The symbol $F_{E}$ will also denote the functor $\mathscr{A}(R) \rightarrow \mathscr{N}(\bar{A})$, restriction of the functor $F_{E}:$ Mod- $R \rightarrow \operatorname{Mod}-\bar{A}$. These functors $F_{E}: \mathscr{A}(R) \rightarrow$ $\mathscr{N}(\bar{A})$ are the traits $d$ 'union between algebraically compact modules and spectral categories. Note that for any fixed cardinal number $\alpha$, we can always choose the module $E$ in such a way that every algebraically compact module $M$ of cardinality $\leq \alpha$ is a direct summand of $E$, and in this case $F_{E}(M)$ is simply a direct summand of the regular, right self-injective ring $\bar{A}$. For these modules $M$ of cardinality $\leq \alpha$ we do not lose any information by applying the functor $F_{E}$, as the next proposition shows.

Proposition 1. Let $E$ be an algebraically compact module such that every algebraically compact module is a direct summand of a power of $E$. Let $M, N$ be direct summands of $E$. Then: 
(a) If $e$ is an idempotent endomorphism of $E$ with image $M$ and $\bar{e} \in \bar{A}$ is the image of $e \in A$, then $F_{E}(M) \cong \overline{e A}$.

(b) $M \cong N$ if and only if $F_{E}(M) \cong F_{E}(N)$.

(c) $M=0$ if and only if $F_{E}(M)=0$.

(d) $M$ is indecomposable if and only if $F_{E}(M)$ is indecomposable.

(e) For every direct summand $K$ of $\bar{A}$ there is a direct summand $P$ of $E$ with $F_{E}(P) \cong K$.

Proof. (a) Trivial.

(b) Suppose $F_{E}(M) \cong F_{E}(N)$. Let $e, f$ be idempotent endomorphisms of $E$ with $e E=M$ and $f E=N$. Let $\bar{e}, \bar{f}$ be their images in $\bar{A}$. Then, by (a), $\overline{e A} \cong \overline{f A}$, so that the projective covers $e A$ and $f A$ of the isomorphic $A$-modules $\overline{e A}$ and $\overline{f A}$ are isomorphic [1, Lemma 27.3]. Tensoring the isomorphism $e A \cong f A$ with the $(A, R)$-bimodule $E$, we get that $e A \otimes_{A} E$ $\cong e E=M$ is isomorphic to $f A \otimes_{A} E \cong f E=N$. The converse is obvious.

(c) Obvious by (b).

(e) Let $K$ be a direct summand of $\bar{A}$. Then $K=\overline{e A}$ for some idempotent $\bar{e}$ of $\bar{A}$. Since idempotents of $\bar{A}$ can be lifted to idempotents of $A, \bar{e}$ is the image of an idempotent $e$ of $A$. Then $P=e E$ satisfies (e) by (a).

(d) If $M \cong M^{\prime} \oplus M^{\prime \prime}$ with $M^{\prime}, M^{\prime \prime} \neq 0$, then $F_{E}(M) \cong F_{E}\left(M^{\prime}\right) \oplus$ $F_{E}\left(M^{\prime \prime}\right)$ because $F_{E}$ is additive, and $F_{E}\left(M^{\prime}\right), F_{E}\left(M^{\prime \prime}\right) \neq 0$ by (c). Therefore if $F_{E}(M)$ is indecomposable, $M$ is indecomposable too. Conversely, if $F_{E}(M)$ is a nontrivial direct sum, then, as in (e), the decomposition of $F_{E}(M)$ can be lifted to a decomposition of $M$ because two orthogonal idempotents in $\bar{A}$ lift to two orthogonal idempotents of $A$.

Corollary 2. ([3], [6, Thm. 3.34] and [21, Lemma 6.6].) If $M, N$ are algebraically compact modules such that each is isomorphic to a pure submodule of the other, then $M \cong N$.

Proof. We may suppose $M, N$ isomorphic to direct summands of $E$ by Corollary 1 . Since $M$ and $N$ are isomorphic to a direct summand of the other, the same happens for $F_{E}(M)$ and $F_{E}(N)$. By [3], $F_{E}(M) \cong F_{E}(N)$. Then $M \cong N$ by Proposition 1(b).

3. Direct sums. Recall that if $M$ is a pure submodule of $N$, then $N$ is a pure-essential extension of $M$ if there are no nonzero submodules $S \subset N$ with $S \cap M=0$ and the image of $M$ pure in $N / S$ [19]. A pure extension $N$ of $M$ is a pure-injective envelope if $N$ is algebraically compact (= pure-injective) and the extension is pure-essential. Pure-injective envelopes exist and are unique up to isomorphism [19, Prop. 6]. We will 
denote a pure-injective envelope of a module $M$ by $\operatorname{PE}(M)$ and an injective envelope of $M$ by $\mathrm{E}(M)$.

The next theorem shows that $F_{E}$ "behaves well" with respect to direct sums. Recall that if $\left\{X_{\lambda} \mid \lambda \in \Lambda\right\}$ is any set of objects of $\mathscr{N}(\bar{A})$, their direct sum in $\mathscr{N}(\bar{A})$ is $\mathrm{E}\left(\oplus_{\lambda \in \Lambda} X_{\lambda}\right)$, the injective envelope of the direct sum in Mod- $\bar{A}$ [11, Prop. 1.12].

THEOREM 3. Let $E$ be an algebraically compact $R$-module such that every $R$-module is isomorphic to a pure submodule of a power of $E$ and let $\left\{M_{\lambda} \mid \lambda \in \Lambda\right\}$ be a family of algebraically compact $R$-modules. Assume that $\oplus_{\lambda \in \Lambda} M_{\lambda}$ is isomorphic to a pure submodule of $E$. Then

$$
F_{E}\left(\operatorname{PE}\left(\bigoplus_{\lambda \in \Lambda} M_{\lambda}\right)\right) \cong \mathrm{E}\left(\bigoplus_{\lambda \in \Lambda} F_{E}\left(M_{\lambda}\right)\right)
$$

Proof. Since $\oplus_{\lambda \in \Lambda} M_{\lambda}$ is isomorphic to a pure submodule of $E$, we may suppose that $\mathrm{PE}\left(\oplus_{\lambda \in \Lambda} M_{\lambda}\right)$ is a direct summand of $E$ [19, Prop. 6]. Let us fix a decomposition $E=\operatorname{PE}\left(\oplus_{\lambda \in \Lambda} M_{\lambda}\right) \oplus C$ and consider the canonical projections of $E$ onto the direct summands of this decomposition; we have idempotents $e$ and $e_{\lambda}, \lambda \in \Lambda$, of $\operatorname{End}\left(E_{R}\right)$ such that $e E=\operatorname{PE}\left(\oplus_{\lambda} M_{\lambda}\right), e_{\lambda} E=M_{\lambda}, e_{\lambda} e=e e_{\lambda}=e_{\lambda}$ for all $\lambda \in \Lambda$, and $e_{\lambda} e_{\mu}=0$ for $\lambda \neq \mu$. Then $F_{E}\left(\operatorname{PE}\left(\oplus_{\lambda} M_{\lambda}\right)\right) \cong \overline{e A}$ and $F_{E}\left(M_{\lambda}\right) \cong \bar{e}_{\lambda} \bar{A}$ by Proposition 1(a), and we must prove that $\overline{e A} \cong \mathrm{E}\left(\oplus_{\lambda} \bar{e}_{\lambda} \bar{A}\right)$. Observe that the submodules $\bar{e}_{\lambda} \bar{A}, \lambda \in \Lambda$, of $\overline{e A}$ are independent because the idempotents $e_{\lambda}$ are pairwise orthogonal; therefore the sum $\sum_{\lambda} \bar{e}_{\lambda} \bar{A} \leq \overline{e A}$ is direct. Since $\bar{A}$ is self-injective, $\overline{e A}$ is injective and contains an injective envelope of $\oplus_{\lambda} \bar{e}_{\lambda} \bar{A}$. Therefore we have $\overline{e A}=\mathrm{E}\left(\oplus_{\lambda} \bar{e}_{\lambda} \bar{A}\right) \oplus S$ for some right $\bar{A}$-module $S$. Let $\pi \in \operatorname{End}_{\bar{A}}(\overline{e A})$ be the projection onto $S$ with kernel $\mathrm{E}\left(\oplus_{\lambda} \bar{e}_{\lambda} \bar{A}\right)$. Since $\operatorname{End}_{\bar{A}}(\overline{e A}) \cong \overline{e A e} \cong e A e / J(e A e)$ [1, Cor. 17.13], the endomorphism $\pi$ corresponds to an idempotent $\bar{f}$ of $e A e / J(e A e)$. Now $e A e \cong \operatorname{End}_{R}(e E)$ is the endomorphism ring of the a.c. module $e E$, so that idempotents can be lifted modulo the Jacobson radical. Let $f$ be an idempotent of $e A e$ whose image is $\bar{f}$. Then $f$ is an idempotent of $A$ and $e f=f e=f$. Note that $\mathrm{E}\left(\oplus_{\lambda} \bar{e}_{\lambda} \bar{A}\right)=\mathrm{ker} \pi$, so $\pi\left(\bar{e}_{\lambda}\right)=0$ for all $\lambda$, i.e. $\overline{f e_{\lambda}}=0$, that is, $f e_{\lambda} \in J(A)$ for all $\lambda \in \Lambda$.

Consider the direct summand $f E=e f E$ of $e E$. Let us show that the map $\varepsilon: \oplus_{\lambda} M_{\lambda} \rightarrow e E / f E$ induced by the inclusion $\oplus_{\lambda} M_{\lambda} \rightarrow e E$ is injective and that the image of $\varepsilon$ is a pure submodule of $e E / f E$. We must prove that $\varepsilon \otimes 1_{M}:\left(\oplus_{\lambda \in \Lambda} M_{\lambda}\right) \otimes_{R} M \rightarrow(e E / f E) \otimes_{R} M$ is injective for every left $R$-module $M$, i.e. we must prove that the image of the mapping 
$\left(\oplus_{\lambda \in \Lambda} M_{\lambda}\right) \otimes M \rightarrow e E \otimes M$ intersects the kernel of the mapping $e E \otimes$ $M \rightarrow(e E / f E) \otimes M$ at zero. The kernel of this second mapping is the image of the canonical embedding $f E \otimes M \rightarrow e E \otimes M$. Therefore it is sufficient to prove that for every finite subset $\left\{\lambda_{1}, \ldots, \lambda_{n}\right\}$ of $\Lambda$ the images of the two canonical embeddings $\left(M_{\lambda_{1}} \oplus \cdots \oplus M_{\lambda_{n}}\right) \otimes M \rightarrow E \otimes M$ and $f E \otimes M \rightarrow E \otimes M$ intersect at zero. Since $f e_{\lambda_{1}}+\cdots+f e_{\lambda_{n}} \in J(A)$, the mapping

$$
\begin{aligned}
\left(f e_{\lambda_{1}}+\cdots+\right. & \left.f e_{\lambda_{n}}-1_{E}\right) \otimes 1_{M} \\
& =\left(f \otimes 1_{M}\right)\left(\left(e_{\lambda_{1}}+\cdots+e_{\lambda_{n}}\right) \otimes 1_{M}\right)-1_{E \otimes M}
\end{aligned}
$$

is an automorphism of $E \otimes M$. But if an element is in the image of $f E \otimes M \rightarrow E \otimes M$, it is fixed by $f \otimes 1_{M}$, and if an element is in the image of $\left(M_{\lambda_{1}} \oplus \cdots \oplus M_{\lambda_{n}}\right) \otimes M$, it is fixed by $\left(e_{\lambda_{1}}+\cdots+e_{\lambda_{n}}\right) \otimes 1_{M}$. Therefore an element in the intersection of the two images is annihilated by the previous automorphism, whence it is zero, as desired.

We have thus proved that $\varepsilon$ is injective, so that $f E$ is a submodule of $e E$ such that $f E \cap\left(\oplus_{\lambda} M_{\lambda}\right)=0$, and the image of $\oplus_{\lambda} M_{\lambda}$ is pure in $e E / f E$. Since $\oplus_{\lambda} M_{\lambda}$ is pure-essential in $e E=\operatorname{PE}\left(\oplus_{\lambda} M_{\lambda}\right)$, we get $f E=0$, $f=0, \bar{f}=0, \pi=0, S=0$ and $\mathrm{E}\left(\oplus_{\lambda} \bar{e}_{\lambda} \bar{A}\right)=\overline{e A}$. This concludes the proof of the theorem.

As a corollary we get the decomposition into discrete and continuous part analogous with $[8, \S 3]$.

COROLlaRy 3 [21, Thm. 6.1]. Every algebraically compact module has a decomposition $M=\operatorname{PE}\left(\oplus_{\lambda \in \Lambda} M_{\lambda}\right) \oplus N$, where the $M_{\lambda}$ 's are indecomposable and $N$ has no indecomposable direct summands. If $M=\operatorname{PE}\left(\oplus_{\lambda^{\prime} \in \Lambda^{\prime}} M_{\lambda^{\prime}}^{\prime}\right)$ $\oplus N^{\prime}$ is another such decomposition, then $N \cong N^{\prime}$ and there is a bijection $\Lambda \rightarrow \Lambda^{\prime}$ such that corresponding indecomposable modules $M_{\lambda}$ and $M_{\lambda^{\prime}}^{\prime}$ are isomorphic.

Proof. Proposition 1, Theorem 3 and [8, §3].

4. Algebraically compact modules as a category. In $\S 2$ we considered the full subcategory $\mathscr{A}(R)$ of Mod- $R$ generated by all algebraically compact $R$-modules, but the results of $\S \S 2$ and 3 and the ones of [8] and [20] lead us to consider another category whose objects are the algebraically compact modules.

Let $f: M \rightarrow N$ be a homomorphism of $R$-modules. It is easy to prove that the following three conditions are equivalent: (i) $f g \in J\left(\operatorname{End}_{R}(N)\right.$ ) for all $g \in \operatorname{Hom}_{R}(N, M)$; (ii) $g f \in J\left(\operatorname{End}_{R}(M)\right.$ ) for all $g \in$ $\operatorname{Hom}_{R}(N, M)$; (iii) $\left(\begin{array}{cc}0 & 0 \\ f & 0\end{array}\right) \in J\left(\operatorname{End}_{R}(M \oplus N)\right)$. (Here $J(S)$ is the Jacobson 
radical of the ring $S$, and homomorphisms of direct sums into direct sums are denoted by matrices.) Therefore the set of all $f \in \operatorname{Hom}_{R}(M, N)$ satisfying these equivalent conditions is a subbimodule of the $(\operatorname{End}(N)$, End $(M))$-bimodule $\operatorname{Hom}_{R}(M, N)$. We denote it by $J\left(\operatorname{Hom}_{R}(M, N)\right)$. Note that, when $M=N, J\left(\operatorname{Hom}_{R}(M, M)\right)$ is exactly the Jacobson radical of the ring $\operatorname{End}_{R}(M)$.

Define the category $\mathscr{C}(R)$ whose objects are all the algebraically compact right $R$-modules with morphism groups

$$
\operatorname{Hom}_{\mathscr{C}(R)}(M, N)=\operatorname{Hom}_{R}(M, N) / J\left(\operatorname{Hom}_{R}(M, N)\right) \text {. }
$$

THeOREM 4. $\mathscr{C}(R)$ is an additive category. The direct sum of a finite number of objects in $\mathscr{C}(R)$ is their direct sum as $R$-modules. Objects of $\mathscr{C}(R)$ are isomorphic in $\mathscr{C}(R)$ if and only if they are isomorphic as modules. Every full small subcategory of $\mathscr{C}(R)$ is equivalent to a full subcategory of a spectral category.

Proof. Most of the proof consists of trivial verifications which we omit. Let us prove the last statement. Given a full small subcategory $\mathscr{D}$ of $\mathscr{C}(R)$ there is a cardinal $\alpha$ such that every module in the subcategory has cardinality $\leq \alpha$. Let $E$ be a module as in Corollary 1 . Let us prove that the functor $F_{E}: \mathscr{A}(R) \rightarrow \mathscr{N}(\bar{A})$ of Theorem 2 induces a faithful full functor $\mathscr{D} \rightarrow \mathscr{N}(\bar{A})$. We must show that if $M, N$ are summands of $E$, the mapping $F_{E}(M, N): \operatorname{Hom}_{R}(M, N) \rightarrow \operatorname{Hom}_{\bar{A}}\left(F_{E}(M), F_{E}(N)\right)$ induced by $F_{E}$ is surjective with kernel $J\left(\operatorname{Hom}_{R}(M, N)\right)$. But if $e, f$ are $R$-endomorphism of $E$ with $M=e E$ and $N=f E$, then $\operatorname{Hom}_{R}(M, N) \cong f A e$ and $\operatorname{Hom}_{\bar{A}}\left(F_{E}(M), F_{E}(N)\right) \cong \operatorname{Hom}_{\bar{A}}(\overline{e A}, \overline{f A}) \cong \overline{f A e}$ canonically.

5. Decompositions of algebraically compact modules. The last result of $\S 3$ was that any algebraically compact module is the direct sum of a discrete part (the pure-injective envelope of a direct sum of indecomposable algebraically compact modules) and a continuous part (an algebraically compact module with no indecomposable direct summands). There is an extensive classification of the objects of a spectral category [11] and the results that hold for any set of objects of a spectral category can be interpreted into the category $\mathscr{C}(R)$ via Theorem 4 . In this section we classify and decompose algebraically compact modules into types. All our results easily follow from our previous theory and [11].

Note that for any algebraically compact module $M$ and for any algebraically compact module $E$ containing $M$ as a direct summand, $\operatorname{End}_{\vec{A}}\left(F_{E}(M)\right) \cong \operatorname{End}_{R}(M) / J\left(\operatorname{End}_{R}(M)\right.$ ) (proof of Theorem 4), and therefore $\operatorname{End}_{\bar{A}}\left(F_{E}(M)\right)$ depends only on $M$ and not on $E$ or $\bar{A}$. 
We say that an algebraically compact right $R$-module $M$ is abelian if the ring $\operatorname{End}_{R}(M) / J\left(\operatorname{End}_{R}(M)\right)$ is abelian [11, Ch. 2], i.e. all its idempotents are central. This happens if and only if the $\bar{A}$-module $F_{E}(M)$ is abelian for any module $E$ containing $M$ as a direct summand and with the properties of Corollary 1 . An algebraically compact $R$-module $M$ is abelian if and only if for every direct sum decomposition $M=M_{1} \oplus M_{2}$ one has $\operatorname{Hom}_{R}\left(M_{1}, M_{2}\right)=J\left(\operatorname{Hom}_{R}\left(M_{1}, M_{2}\right)\right)$ [11, Thm. 2.1].

In analogy with [11, Ch. 3], we say that an algebraically compact module $M$ is directly finite if the ring $\operatorname{End}_{R}(M) / J\left(\operatorname{End}_{R}(M)\right)$ is directly finite, i.e. all its one-sided inverses are two-sided. If $E$ is any algebraically compact module with the properties of Corollary 1 and "big enough", that is, containing $M$ as a direct summand, then $M$ is directly finite if and only if the $\bar{A}$-module $F_{E}(M)$ is directly finite. It is easy to see as in [11, Thm. 3.1] that an algebraically compact $R$-module $M$ is directly finite if and only if $M$ is not isomorphic to any proper direct summand of itself, or, equivalently, if $M$ has no nonzero direct summands $N$ with $N \oplus N \cong N$.

Let $M$ be an algebraically compact module. Then we say that:

(i) $M$ is Type I if every nonzero direct summand of $M$ has a nonzero abelian direct summand;

(ii) $M$ is Type II if every nonzero direct summand of $M$ has a nonzero directly finite summand but $M$ has no nonzero abelian direct summands;

(iii) $M$ is Type III if it has no nonzero directly finite direct summands;

(iv) $M$ is purely infinite if $M \oplus M \cong M[11, \mathrm{Ch} .5$ and 6].

Then an algebraically compact module $M$ is Type I, II, III or purely infinite if and only if $\operatorname{End}_{R}(M) / J\left(\operatorname{End}_{R}(M)\right)$ is Type I, II, III or purely infinite, or, equivalently, if and only if the nonsingular injective $\bar{A}$-module $F_{E}(M)$ is Type I, II, III or purely infinite ( $E, A$ and $\bar{A}$ as above).

For instance every ring $R$ has algebraically compact modules of Type I: if $S$ is a simple $R$-module, then $\mathrm{E}(S)$ is an indecomposable injective $R$-module, and $a$ fortiori an algebraically compact abelian module.

Finally, we say that a module $M$ is Type $\mathrm{I}_{\mathrm{f}}$ if $M$ is Type $\mathrm{I}$ and directly finite; Type $\mathrm{I}_{\infty}$ if $M$ is Type $\mathrm{I}$ and purely infinite; Type $\mathrm{II}_{\mathrm{f}}$ if $M$ is Type II and directly finite; Type $\mathrm{II}_{\infty}$ if $M$ is Type II and purely infinite.

TheOREM 5 [11, Cor. 7.6]. Any algebraically compact right $R$-module is a direct sum of five modules of Types $\mathrm{I}_{\mathrm{f}}, \mathrm{I}_{\infty}, \mathrm{II}_{\mathrm{f}}, \mathrm{II}_{\infty}, \mathrm{III}$, respectively, uniquely up to isomorphism of the direct summands.

6. Dimension theories and other applications. In the mathematical literature many cardinal invariants have been defined for algebraically compact modules: Ziegler defined the dimensions $\operatorname{dim}_{U}$, where $U$ is a 
module with local endomorphism ring [21, §6]; Loś and Kaplansky's complete description of the algebraically compact abelian groups ([12] and [13]) leads to a natural definition of a family of cardinals $\alpha_{0}, \alpha_{p}, \beta_{p, n}$ and $\gamma_{p}$, where $p$ ranges in the prime numbers and $n$ in the natural numbers, and these cardinals form a complete set of invariants [16, Ch. IV, 1.6]; Warfield constructed complete sets of invariants for particular algebraically compact modules over Pfüfer domains [19].

On the other hand there is a complete dimension theory for the objects of a spectral category [11]. In this section we are going to examine the relations between these various dimension theories. The bridge that allows us to pass from one dimension theory to the others is constituted by the functors $F_{E}$.

Let us begin by studying the relation between the dimension theory constructed by Goodearl and Boyle and that of Ziegler.

Let $C, U$ be algebraically compact modules and suppose $U$ indecomposable. By Corollary $3, C$ has a decomposition $C=\operatorname{PE}\left(\oplus_{\lambda \in \Lambda} U_{\lambda}\right) \oplus D$, where each $U_{\lambda}$ is indecomposable and algebraically compact, and $D$ has no indecomposable direct summands. Ziegler [21, §6] defined the dimension of $C$ relative to $U$ as $\operatorname{dim}_{U}(C)=\left\{\left\{\lambda \in \Lambda \mid U_{\lambda} \cong U\right\} \mid\right.$. If we apply the functor $F_{E}$ to the above decomposition, where $E$ is an algebraically compact module containing $C$ and $U$ as direct summands and with the property that every module is isomorphic to a pure submodule of a power of $E$, we get that $F_{E}(C) \cong \mathrm{E}\left(\oplus_{\lambda \in \Lambda} F_{E}\left(U_{\lambda}\right)\right) \oplus F_{E}(D)$ (Theorem 3). Since $U$ is an indecomposable direct summand of $E, U=u E$ for some primitive idempotent $u$ of $A=\operatorname{End}(E)$, and if $\bar{u} \in \bar{A}=A / J(A)$ is its image, then $\bar{u}$ is primitive because idempotents lift modulo $J(A)$. Therefore the set of all central idempotents $\bar{e}$ of $\bar{A}$ that annihilate $\bar{u}$ (i.e. $\overline{e u}=0$ ) is a maximal ideal $M$ of the Boolean algebra $B(\bar{A})$ of all central idempotents in $\bar{A}$. (Notations as in [11, Ch. IV].) Moreover, if $\operatorname{cc}(\bar{u})$ denotes the central cover of $\bar{u}$, i.e. the smallest central idempotent in $\bar{A}$ which acts as the identity on $\bar{u}$, then $M$ is the ideal of $B(\bar{A})$ generated by $1-\operatorname{cc}(\bar{u})$. Note that $M$ is an isolated point in the Boolean spectrum $\operatorname{BS}(\bar{A})$ of $\bar{A}$, that is, the space of all maximal ideals in $B(\bar{A})$ (notations as in [11, Ch. IX]). Therefore the $\bar{A}$-module $F_{E}(C) \operatorname{cc}(\bar{u})$ is the injective envelope, as an $\bar{A}$-module, of the direct sum of all the $F_{E}\left(U_{\lambda}\right)$ 's with $U_{\lambda} \cong U, \lambda \in \Lambda$. It is now clear that Goodearl's and Boyle's invariant $d_{M}\left(F_{E}(C): F_{E}(U)\right)$ indexed in the point $M$ of the compact, totally disconnected Hausdorff space $\operatorname{BS}(\bar{A})$ is equal to Ziegler's invariant $\operatorname{dim}_{U}(C)$, if this is finite, and equal to $\infty$, otherwise. Similarly Goodearl's and Boyle's invariant $\mu_{M}(C)$ is 0 if Ziegler's invariant $\operatorname{dim}_{U}(C)$ is 0 , and is the smallest infinite cardinal greater than 
$\operatorname{dim}_{U}(C)$, otherwise. This is the connection between Goodearl's and Boyle's dimension theory and Ziegler's dimension theory.

Let us pass to consider algebraically compact abelian groups. (Here abelian group means $Z$-module as usual and there is no connection with abelian algebraically compact modules as defined in the previous section.) An abelian group is algebraically compact if and only if it is the direct sum of a divisible group and a group complete in the $Z$-adic topology [7, Thm. 39.1]. The indecomposable algebraically compact groups up to isomorphism are $Z\left(p^{n}\right)$ (the cyclic group of order $p^{n}, p$ a prime), $Z\left(p^{\infty}\right)$ (Prüfer's quasicyclic group), $Q$ (the group of rationals) and $J_{p}$ (the group of $p$-adic integers, $p$ a prime) [7, Cor. 40.4].

Proposition 2. Let $S=\left\{Z\left(p^{n}\right), Z\left(p^{\infty}\right), Q, J_{p} \mid p\right.$ prime, $\left.n \geq 1\right\}$ be an irredundant set of representatives of all indecomposable algebraically compact groups. Let $E$ be any algebraically compact group such that every element of $S$ is isomorphic to a direct summand of $E$. Then the Boolean algebra $B(\operatorname{End}(E) / J(\operatorname{End}(E)))$ of all central idempotents in $\operatorname{End}(E) / J(\operatorname{End}(E))$ is isomorphic to the Boolean algebra of all subsets of the set $S$.

Proof. Set $A=\operatorname{End}(E)$ and $\bar{A}=A / J(A)$, and consider the $\bar{A}$-modules $F_{E}(G), G \in S$. By Proposition $1(\mathrm{~d})$ they are indecomposable injective summands of $\bar{A}$, and therefore they are simple pairwise nonisomorphic modules. Since every a.c. group has a direct summand isomorphic to an element of $S$ [7, Cor. 40.4], the set $\left\{F_{E}(G) \mid G \in S\right\}$ generates the category $\mathscr{N}(\bar{A})$. In particular, the center of $\bar{A}$ and the center of End ${ }_{\mathscr{N}(\bar{A})}\left(\oplus_{G \in S} F_{E}(G)\right)$, where the direct sum is in $\mathscr{N}(\bar{A})$, are isomorphic. But the $F_{E}(G)$ 's are simple and pairwise nonisomorphic, so that the endomorphism ring of their direct sum is a direct product of division rings indexed in $S$. Therefore $B(\bar{A})$ is isomorphic to the Boolean algebra of all subsets of the set $S$.

It follows that the spectrum of $B(\operatorname{End}(E) / J(\operatorname{End}(E)))$ is homeomorphic to the Stone-Čech compactification of the set $S$ with the discrete topology. In particular, suppose $E=\operatorname{PE}\left(\oplus_{G \in S} G\right)$. Then for each algebraically compact group $C$, Goodearl's and Boyle's continuous function $d_{-}(C: E)$ from the Stone-Čech compactification of the set $S$ into the extended interval $[0,+\infty]$ is determined by its restriction to the set $S$. This restriction to $S$ is the map $S \rightarrow[0,+\infty]$ which assigns to each group $G \in S$ the corresponding invariant of the group $C$ as defined in Orsatti's 
book [16, Cap. IV, §1.6]. Here "corresponding" means the invariant $\alpha_{0}(C)$ if $G=Q, \alpha_{p}(C)$ if $G=Z\left(p^{\infty}\right), \beta_{p, n}(C)$ if $G=Z\left(p^{n}\right)$, and $\gamma_{p}(C)$ if $G=J_{p}$ (and if these invariants are infinite cardinals, we assign them $\infty$ ). Similarly Goodearl's and Boyle's invariant $\mu_{-}(C)$ is the "continuous" (in the sense of $[5, \S 7])$ extension of the map of $S$ into the class Card of all cardinal numbers, which assigns to each group $G \in S$ either the smallest infinite cardinal greater than the corresponding invariant of the group $C$ as defined in Orsatti's book, or zero (if Orsatti's invariant is zero.)

Let us conclude with some remarks concerning Warfield's decomposition of algebraically compact modules over Prüfer domains. The module $E$ of the proof of Theorem 1 (and all its powers $E^{\alpha}$ ) are duals of direct sums of finitely generated modules. If $R$ is a Prüfer domain every finitely generated module is the direct sum of its torsion part and a projective module [19, Prop. 5]. From this it follows that $E^{\alpha}$ is the direct sum of a module $M_{1}$ with no elements of infinite height (that is, no nonzero element divisible by all nonzero elements of $R$ ) and an injective module $M_{2}$. Since $M_{2}$ is divisible, $\operatorname{Hom}_{R}\left(M_{2}, M_{1}\right)=0$, so that $\operatorname{Hom}_{R}\left(M_{1}, M_{2}\right)=$ $J\left(\operatorname{Hom}_{R}\left(M_{1}, M_{2}\right)\right)(\S 4)$. It follows that the Boolean spectrum of $E^{\alpha}$ is the disjoint union of two clopen sets, the first corresponding to the algebraically compact modules with no elements of infinite height and the second corresponding to the injective modules. This is the decomposition of [19, Prop. 11].

Let us keep the same notation, but let us suppose that the Prüfer domain $R$ is $h$-local. Then a torsion module is the direct sum of its localizations [15]. Thus $M_{1}$, dual of a torsion module, is the direct product of its localizations. Therefore in the Boolean spectrum of $E^{\alpha}$ the clopen set corresponding to the algebraically compact modules with no elements of infinite height is the Stone-Čech compactification of a disjoint union of clopen sets, one for each maximal ideal of $R$. This corresponds to the decomposition of [19, Prop. 12].

\section{REFERENCES}

[1] F. W. Anderson and K. R. Fuller, Rings and Categories of Modules, Berlin, Springer, 1974.

[2] S. Balcerzyz, On algebraically compact groups of I. Kaplansky, Fund. Math., 44 (1957), 91-93.

[3] R. T. Bumby, Modules which are isomorphic to submodules of each other, Arch. Math., 16 (1965), 184-185.

[4] F. Couchot, Sous-Modules Purs et Modules de Type Cofini, Séminaire d'Algèbre Paul Dubreil, Proceedings, Paris 1976-1977, Lecture Notes in Math. 641, Berlin, Springer (1978), 198-208. 
[5] A. Facchini, Spectral categories and varieties of preadditive categories, J. Pure Appl. Algebra, 29 (1983), 219-239.

[6] E. R. Fisher, Abelian Structures I, Abelian Group Theory, 2nd New Mexico State University Conference, 1976, Lecture Notes in Math. 616, Berlin, Springer (1977), 270-322.

[7] L. Fuchs, Infinite Abelian Groups, Vol. I, New York, Academic Press, 1970.

[8] P. Gabriel and U. Oberst, Spektralkategorien und reguläre Ringe im VonNeumannschen Sinn, Math. Z., 92 (1966), 389-395.

[9] S. Garavaglia, Direct product decomposition of theories of modules, J. Symbolic Logic, 44 (1979), 77-88.

[10] , Decomposition of totally transcendental modules, J. Symbolic Logic, 45 (1980), 155-164.

[11] K. R. Goodearl and A. K. Boyle, Dimension theory for nonsingular injective modules, Mem. Amer. Math. Soc., 177 (1976).

[12] I. Kaplansky, Infinite abelian groups, Ann Arbor, Univ. of Michigan Press, 1954.

[13] J. Loś, Abelian groups that are direct summands of every abelian group which contains them as pure subgroups, Fund. Math., 44 (1957), 84-90.

[14] J. M. Maranda, On pure subgroups of abelian groups, Arch. Math., 11 (1960), 1-13.

[15] E. Matlis, Cotorsion Modules, Mem. Amer. Math. Soc., 49 (1964).

[16] A. Orsatti, Introduzione ai gruppi abeliani astratti e topologici, Bologna, Pitagora Ed., 1979.

[17] J. E. Roos, Locally distributive spectral categories and strongly regular rings, Reports of the Midwest Category Seminar, Lecture Notes in Math. 47, Berlin, Springer (1967), 156-181.

[18] B. T. Stenström, Pure submodules, Ark. Mat., 7 (1969), 159-171.

[19] R. B. Warfield, Purity and algebraic compactness for modules, Pacific J. Math., 28 (1969), 699-719.

[20] , Decompositions of injective modules, Pacific J. Math., 31 (1969), 263-276.

[21] M. Ziegler, Model theory of modules, Ann. Pure Appl. Logic, 26 (1984), 149-213.

[22] B. Zimmermann-Huisgen and W. Zimmermann, Algebraically compact rings and modules, Math. Z., 161 (1978), 81-93.

Received March 3, 1983 and in revised form December 14, 1983.

UNIVERSITA DI UDINE

33100 UDINE, ITALY 



\section{PACIFIC JOURNAL OF MATHEMATICS \\ EDITORS}

Donald BABBITT (Managing Editor)

University of California

Los Angeles, CA 90024

J. Dugundu

University of Southern Californa

Los Angeles, CA 90089-1113

R. FINN

Stanford University

Stanford, CA 94305

HermanN FLaSChKa

University of Arizona

Tucson, AZ 85721

\author{
C. C. MOORE \\ University of California \\ Berkeley, CA 94720 \\ ARTHur Ogus \\ University of California \\ Berkeley, CA 94720 \\ Hugo Rossi \\ University of Utah \\ Salt Lake City, UT 84112 \\ H. SAMELSON \\ Stanford University \\ Stanford, CA 94305
}

ASSOCIATE EDITORS
R. ARENS
E. F. BECKENBACH
B. H. NeUmanN
F. WOLF
K. YOSHIDA (1906-1982)

\section{SUPPORTING INSTITUTIONS}

UNIVERSITY OF ARIZONA

UNIVERSITY OF BRITISH COLUMBIA

UNIVERSITY OF OREGON

CALIFORNIA INSTITUTE OF TECHNOLOGY

UNIVERSITY OF CALIFORNIA

MONTANA STATE UNIVERSITY

UNIVERSITY OF SOUTHERN CALIFORNIA

UNIVERSITY OF NEVADA, RENO

STANFORD UNIVERSITY

UNIVERSITY OF HAWAII

NEW MEXICO STATE UNIVERSITY

UNIVERSITY OF TOKYO

UNIVERSITY OF UTAH

WASHINGTON STATE UNIVERSITY

OREGON STATE UNIVERSITY

UNIVERSITY OF WASHINGTON 


\section{Pacific Journal of Mathematics}

\section{Vol. 116, No. $1 \quad$ November, 1985}

K. Adachi, Le problème de Lévi pour les fibrés grassmanniens et les variétés

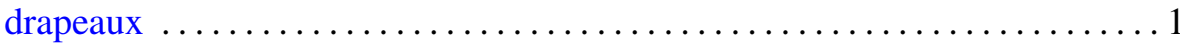

John MacLeod Ball, Remarks on the paper: "Basic calculus of variations" . . . 7 John Kelly Beem and Phillip E. Parker, Whitney stability of solvability . . . 11 Alberto Facchini, Decompositions of algebraically compact modules .......25

S. S. Khare, Finite group action and equivariant bordism $\ldots \ldots \ldots \ldots . \ldots 39$

Horst Leptin, A new kind of eigenfunction expansions on groups $\ldots \ldots \ldots . .45$

Pei-Kee Lin, Unconditional bases and fixed points of nonexpansive

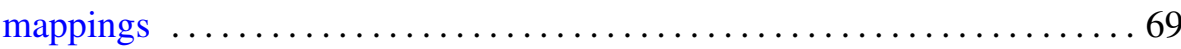

Charles Livingston, Stably irreducible surfaces in $S^{4} \ldots \ldots \ldots \ldots \ldots 77$

Kevin Mor McCrimmon, Nonassociative algebras with scalar involution . . .885

Albert Milani, Singular limits of quasilinear hyperbolic systems in a

bounded domain of $\mathbf{R}^{3}$ with applications to Maxwell's equations

Takemi Mizokami, On $M$-structures and strongly regularly stratifiable

spaces

Jesper M. Møller, On the homology of spaces of sections of complex

projective bundles

Nikolaos S. Papageorgiou, Carathéodory convex integrand operators and

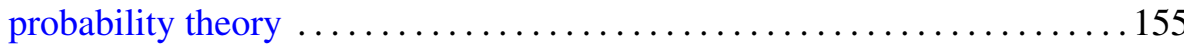

Robert John Piacenza, Transfer in generalized prestack cohomology 185

Lance W. Small and Adrian R. Wadsworth, Integrality of subrings of matrix rings ...

James Michael Wilson, On the atomic decomposition for Hardy spaces 